\title{
Importance of methodological details in the measurement of cortisol in human hair
}

\author{
Lucia BAlagova, Daniela Jezova
}

Laboratory of Pharmacological Neuroendocrinology, Department of Endocrine Regulations and Psychopharmacology, Institute of Experimental Endocrinology, Biomedical Research Center, Slovak Academy of Sciences, Bratislava, Slovakia E-mail:daniela.jezova@savba.sk

\begin{abstract}
Objective. The measurement of cortisol in hair became a popular and frequently used methodology in human stress research. This methodological approach, depending on the length of hair analyzed, allows to reflect cortisol secretion over prolong time periods in a retrospective fashion. There is a big variability in the experimental approaches to cortisol extraction used in individual laboratories. Moreover, there are many methodological details which are not described in most of the published papers, although they may be influential. The aim of the present study was to identify and optimize selected methodological steps of hair cortisol extraction.

Methods. As the starting point served the methodology of Xiang et al. (2016). A hair pool was used to test the procedures. The main steps modified were pulverization, methanol extraction and centrifugation.

Results. In the presented procedure, we decreased the speed and duration of the pulverization, we increased the volume of methanol and increased the time and speed of centrifugation. The results showed obtaining lower variability and higher cortisol concentrations than those we obtained by the methodology of Xiang et al. (2016), which was optimized.

Conclusion. The presented methodology is relatively simple and is likely to provide reliable results with low variability of cortisol concentrations measured in the same sample.
\end{abstract}

Key words: cortisol extraction, human hair, method

Cortisol is the main glucocorticoid hormone in humans and it plays an important role in many physiological functions as well as in pharmacological treatments. A big attention is given to measurement of cortisol release during stress. There are also multiple clinical conditions, which require analysis of cortisol in biological fluids (Nunes et al. 2015). In the past, cortisol concentrations were measured mainly in blood serum or plasma. To avoid the stressfulness of venipuncture, determination of cortisol in saliva is recently often preferred (Jezova and Hlavacova 2008).

Since 2004 (Raul et al. 2004), the measurement of cortisol in hair became a popular and frequently used approach in human stress research. It is considered a suitable methodology particularly for evaluation of long term presence of chronic stress conditions. Hair grows approximately $1 \mathrm{~cm}$ per month (Wennig 2000). Therefore, concentration of cortisol measured in $1 \mathrm{~cm}$ long hair (starting from the scalp) reflects a cumulative value for cortisol secretion during the last month. The hair sampling does not require a specially educated staff and it can be done non-invasively at any time of the day. Moreover, the hair samples can easily by transported and stored at room temperature (Gow et al. 2010).

There are several commercially available kits for measurement of cortisol by radioimmunoassay or enzyme-linked immunosorbent assay (ELISA). Par-

Corresponding author: prof. Daniela Jezova, PharmD., Institute of Experimental Endocrinology, Biomedical Research Center, Slovak Academy of Sciences, Dubravska cesta 9, 84505 Bratislava, Slovakia; phone: 00421259302 404; e-mail: daniela.jezova@savba.sk. 
ticularly the analysis by ELISA kits is relatively simple and may be performed even by people without deep laboratory experience. This is however different with respect to the preparation of the sample and extraction of cortisol from the hair. The importance of laboratory expertise needed for proper hormone extraction is often neglected.

There is a big variability in the experimental approaches to cortisol extraction used in individual laboratories. In the last 10 years, there are more than 300 papers published on measurement of hair cortisol, which differ in the amount of hair taken into extraction, the way of hair cutting or pulverization, duration of extraction or way of extract evaporation. More importantly, there are many methodological details which are not described in most of the published papers, although they may be influential. These include details on washing method, timing and extend of sample preparation or concrete information on centrifugation conditions.

The aim of the present study was to identify and optimize selected methodological steps of hair cortisol extraction. As a starting point we have used one of the few papers providing detailed description of methodology by Xiang and colleagues (2016). These authors compared the suitability of three methodological steps and revealed that it is better to pulverize than cut the hair, to perform the extraction at room temperature or $50^{\circ} \mathrm{C}$ and evaporation of organic solvent by room air than by a stream of nitrogen.

\section{Methods and results}

The amounts of hair for extraction were 12.5, 25 and $50 \mathrm{mg}$. The present study was purely methodological, human hair pool was used without measurement of cortisol concentrations of concrete individuals. According to Xiang et al. (2016) the hair sample was washed 3 times with $5 \mathrm{ml}$ aliquots of isopropyl alcohol (Serva, Germany), dried overnight and pulverized with ULTRA-TURRAX Tube Drive (IKA ${ }^{\circ}$ Works, Inc., Wilmington, NC) at 4000 rounds per minute (rpm) for $6 \mathrm{~min}$ followed by $6000 \mathrm{rpm}$ for 6 min. The BMT-20 tubes contained 40 steel balls. The hair powder was eluted with $1.5 \mathrm{ml}$ of methanol (Sigma-Aldrich, USA) in glass tubes on a rotating shaker at room temperature overnight, centrifuged at 1000 rpm for $2 \mathrm{~min}$ and $1 \mathrm{ml}$ of supernatant was transferred in a new glass tube. Methanol was evaporated at $50^{\circ} \mathrm{C}$ (Xiang et al. 2016) in a water bath. The residue was diluted in $250 \mu \mathrm{l}$ of $0.01 \mathrm{M}$ phosphate buffer saline $(\mathrm{pH} 7.4)$ and $50 \mu \mathrm{l}$ per well was used for the analysis. Cortisol concentrations in hair extracts
Table 1

The high variability of cortisol concentrations in human hair measured in parallel samples before the present modification of the methodology

\begin{tabular}{lccc}
\hline Sample & $\begin{array}{c}\text { Amount of hair } \\
(\mathbf{m g})\end{array}$ & $\begin{array}{c}\text { Cortisol } \\
(\mathbf{n g} / \mathbf{1 0 0} \mathbf{~ m l})\end{array}$ & $\begin{array}{c}\text { Cortisol } \\
(\mathbf{p g} / \mathbf{m g} \text { of hair })\end{array}$ \\
\hline $\mathbf{1}$ & \multirow{2}{*}{12.5} & 36 & 10.47 \\
& & 7 & 2.04 \\
$\mathbf{2}$ & 25 & 69 & 10.45 \\
& & 23 & 3.48 \\
$\mathbf{3}$ & \multirow{2}{*}{50} & 79 & 6.44 \\
& & 55 & 4.48 \\
$\mathbf{4}$ & \multirow{2}{*}{12.5} & 62 & 16.49 \\
& & 28 & 7.45 \\
$\mathbf{5}$ & \multirow{2}{*}{25} & 54 & 7.97 \\
& & 56 & 8.27 \\
$\mathbf{6}$ & 50 & 89 & 7.01 \\
& & 167 & 13.18 \\
\hline
\end{tabular}

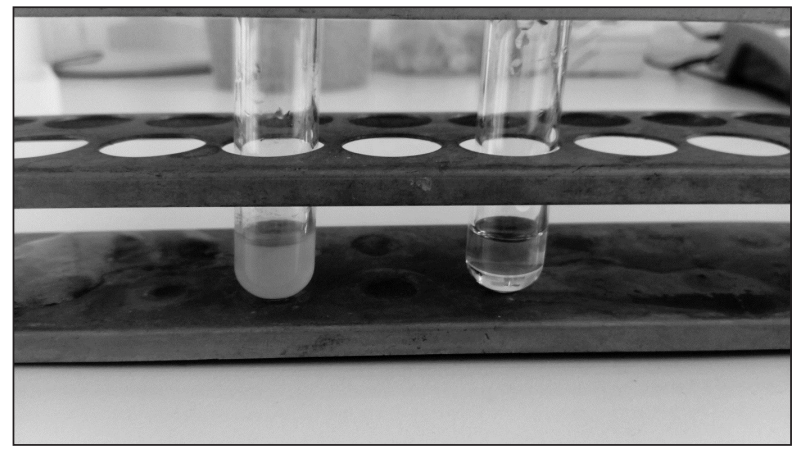

Figure 1. Examples of methanol extract of human hair following centrifugation. The second, clear sample was performed using present methodology.

were measured by the Salivary Cortisol Enzyme Immunoassay (ELISA) kit (IBL International, Hamburg, Germany).

We used the above mentioned methodology (Xiang et al. 2016) on a test hair extraction and we obtained values of cortisol concentrations in appropriate range of the calibration curve. However, the duplicate tubes from the same hair extracts were very variable (Table 1).

Moreover, it happened repeatedly that the methanol extract of hair was cloudy (Figure 1). Next, it was uneasy to pipette the supernatant into the new tube without indirectly disturbing the sediment. Thus, an idea raised that the centrifugation efficacy was not sufficient. We therefore increased the volume of methanol for extraction from 1.5 to $2.0 \mathrm{ml}$ and following centrifugation at $2000 \mathrm{rpm}$ for $10 \mathrm{~min}$ we transferred $1.5 \mathrm{ml}$ of supernatant into an Eppendorf 
Table 2

Cortisol concentrations measured in human hair pool by two methodologies of hair cortisol extraction

\begin{tabular}{|c|c|c|}
\hline Cortisol & $\begin{array}{l}\text { Methodology according } \\
\text { to Xiang et al. (2016) } \\
\text { A }\end{array}$ & $\begin{array}{c}\text { Present methodology } \\
\text { B }\end{array}$ \\
\hline \multirow{10}{*}{ 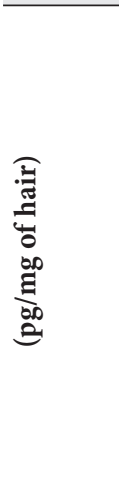 } & 4.1 & 6.0 \\
\hline & 2.5 & 5.2 \\
\hline & 4.8 & 4.9 \\
\hline & 3.1 & 5.8 \\
\hline & 5.1 & 6.3 \\
\hline & 4.8 & 5.3 \\
\hline & 3.6 & 5.5 \\
\hline & 3.8 & 5.3 \\
\hline & 3.9 & 5.9 \\
\hline & 4.5 & 4.8 \\
\hline Mean & 4.01 & $5.49(\mathrm{p}<0.001)$ \\
\hline SD & 0.77 & 0.48 \\
\hline $\mathrm{SD}^{2}$ & 0.59 & 0.23 \\
\hline
\end{tabular}

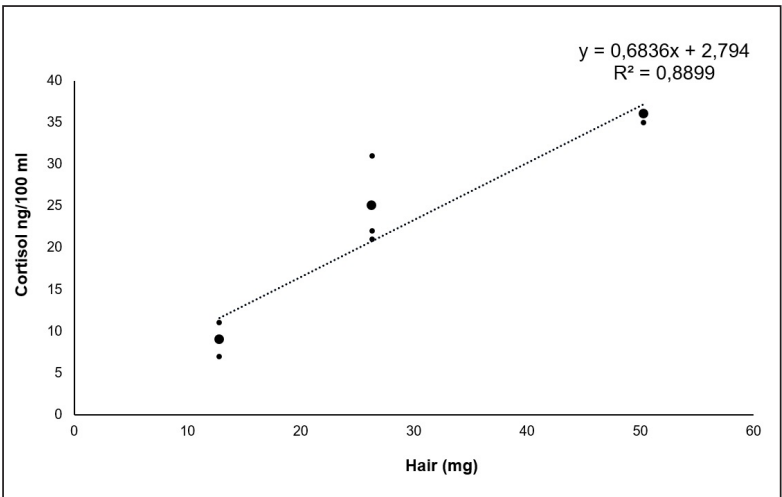

Figure 2. The relationship between the amount of hair pool analyzed and cortisol concentrations obtained.

tube and centrifuged again at $10000 \mathrm{rpm}$ for $10 \mathrm{~min}$. The variability of duplicate samples from the same hair extract was lower, though not ideal.

The next step was to test different speeds and durations of pulverization of hair and their combinations. The results were statistically evaluated using the independent Student t-test. Differences were considered significant at a $\mathrm{p}<0.05$. The linear regression analysis was used to study the linear relationship between different amounts of hair pool and cortisol concentration.

The modified methodology of hair cortisol extraction. Wash the hair sample 3 times with $5 \mathrm{ml}$ aliquots for 2 min of isopropyl alcohol, dry overnight and pulverize with ULTRA-TURRAX Tube Drive (IKA ${ }^{\circ}$ Works, Inc., Wilmington, NC) at $4000 \mathrm{rpm}$ for 6 min. The BMT-20 tubes contained 40 steel balls. Elute the hair powder $(50 \mathrm{mg})$ with $2.0 \mathrm{ml}$ of methanol on a rotating shaker at room temperature overnight, centrifuge at $2000 \mathrm{rpm}$ for $10 \mathrm{~min}$, transfer $1.5 \mathrm{ml}$ of supernatant into an Eppendorf tube and centrifuge again at $10000 \mathrm{rpm}$ for $10 \mathrm{~min}$. Transfer $1.0 \mathrm{ml}$ of methanol to a glass tube, evaporate at $50^{\circ} \mathrm{C}$ (water bath) with room air overnight and dilute with $250 \mu \mathrm{l}$ of $0.01 \mathrm{M}$ phosphate buffer saline (pH 7.4). The sample is ready for cortisol analysis by ELISA as described above.

To compare the above described methodology with the method of Xiang et al. (2016), we performed the washing step with a $200 \mathrm{mg}$ of hair pool and the pulverization at $4000 \mathrm{rpm}$ for $6 \mathrm{~min}$. Thereafter, $50 \mathrm{mg}$ of the powder was transferred to a glass tube for further extraction with $2 \mathrm{ml}$ methanol (sample B). We continued to pulverize the rest of the hair powder by $6000 \mathrm{rpm}$ for $6 \mathrm{~min}$ (Xiang et al. 2016) and $50 \mathrm{mg}$ of the powder was transferred to a glass tube for further extraction with $1.5 \mathrm{ml}$ methanol (sample A). The elution as well as first centrifugation was $1000 \mathrm{rpm}$ for 2 min (sample A) or $2000 \mathrm{rpm}$ for $10 \mathrm{~min}$ (sample B). Sample B was centrifuged again in an Eppendorf tube at $10000 \mathrm{rpm}$ for $10 \mathrm{~min}$. The evaporation step was performed under the same conditions.

The results showed significantly higher cortisol concentrations $(t=4.93 ; \mathrm{p}<0.001)$ in the sample $B$ analyzed by the presently proposed methodology (Table 2). Moreover, the variability of cortisol concentrations measured in the same sample in 10 parallel wells as shown by the values of standard deviation (SD) and squared SD was much lower in sample B.

To evaluate the relationship between the amount of hair pool analyzed and cortisol concentrations obtained, we performed the extraction using the presented methodology from 12.8, 26.3 and $50.3 \mathrm{mg}$. The cortisol assay was performed in triplicates. The results showed a clear linear regression (Figure 2). The coefficient of variation was 0.89 .

\section{Discussion}

The main result of the present studies is the optimization of the methodology for extraction of cortisol from human hair. The important modifications were made in the pulverization and centrifugation steps (Figure 3).

The extraction of cortisol from hair starts with organic solvent. The majority of previously described procedures involves isopropyl alcohol. In many ar- 
ticles, this step is not even mentioned (e.g. Karlen et al. 2011; Caparros-Gonzalez et al. 2017; Pochigaeva et al. 2017). Very variable duration of drying the hair after the washing was used in previous reports. In our hands, the optimal way is to dry the hair for longer time periods, the best is overnight.

There is a big diversity in the step of the hair preparation for extraction. The simplest technology is to mince the hair by scissors (e.g. Smy et al. 2016). To obtain extra fine powder, there is a possibility to pulverize the hair by using a ball mill device (e.g. Laudenslager et al. 2011; Skoluda et al. 2012; Krumbholz et al. 2018), which is rather expensive and not available for each laboratory. We used a middle way, namely the pulverization with a ball grinder. Interestingly, there was not a linear correlation between the length of grinding and the speed of the mixing with reliable results of cortisol analysis. In opposite, the best results were obtained with the lowest speed and the shortest duration of pulverization tested.

A rather uniform step described in the published studies is the extraction of cortisol by methanol. The individual methodologies differ only in the extraction temperature. A very convenient process is to perform the extraction at room temperature, which was used also in the present procedure.

The critical step is the centrifugation of the extracts. The details of the centrifugation conditions are usually not described in the published papers. We observed that the results of final cortisol analysis can be significantly influenced by insufficient centrifugation. Some authors used only slow rotation, e.g. $100 \mathrm{rpm}$ (Albar et al. 2013). The present results are in favor of prolonged (2 times $10 \mathrm{~min}$ ) and rapid (2000 and $10000 \mathrm{rpm}$ ) centrifugation. Such high speed centrifugation has been described only rarely (Kirschbaum et al. 2009; Meyer et al. 2014). It is possible, although we have no direct evidence that after very intensive pulverization, the smallest parts of hair powder may occasionally be transferred into the wells for ELISA analysis. Thus, an insufficient centrifugation along with very intensive pulverization may be the cause of the variability in parallel samples. It cannot be excluded that the variability observed in the mass density of hair (Kiazy-

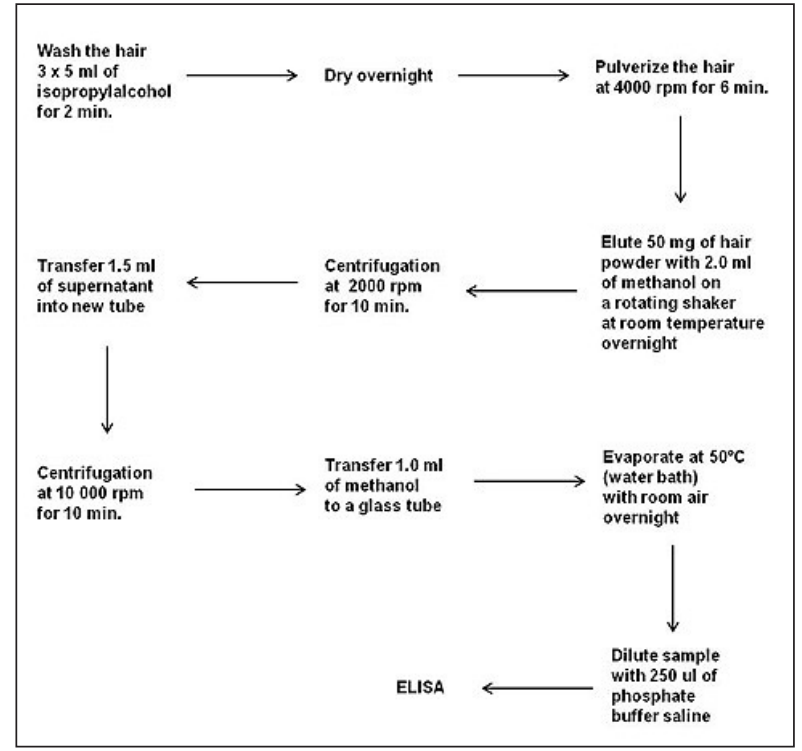

Figure 3. The simplified/schematic steps of the final modified method used for cortisol extraction from human hair.

mov et al. 2009) may contribute to the difficulties mentioned.

Evaporation of methanol before cortisol analysis used to be performed by variable approaches often with necessity of the source of nitrogen stream or speed-vac. Present data are in favor of a simple evaporation with room air as suggested by Xiang et al. (2016), but at a higher temperature $\left(50^{\circ} \mathrm{C}\right)$ using a water bath.

In conclusion, we have optimized several methodological steps in the process of the hair cortisol extraction. The presented methodology is relatively simple and is likely to provide reliable results with low variability of cortisol concentrations measured in the same sample.

\section{Acknowledgements}

The study was supported by the grant of Slovak Research and Development Agency under the contract APVV-15-0063 and of VEGA 2/0057/15. The authors wish to thank Peter Karailiev for helpful suggestions.

\section{References}

Albar WF, Russell EW, Koren G, Rieder MJ, van Umm SH. Human hair cortisol analysis: comparison of the internationally-reported ELISA methods. Clin Invest Med 36, 312-316, 2013.

Caparros-Gonzalez RA, Romero-Gonzalez B, Strivens-Vilchez H, Gonzalez-Perez R, Martinez-Augustin O, PeraltaRamirez MI. Hair cortisol levels, psychological stress and psychopathological symptoms as predictors of postpartum depression. PLoS One 12, e0182817, 2017. 
Gow R, Thomson S, Rieder M, Van Uum S, Koren G. An assessment of cortisol analysis in hair and its clinical applications. Forensic Sci Int 196, 32-37, 2010.

Jezova D, Hlavacova. Endocrine factors in stress and psychiatric disorders: focus on anxiety and salivary steroids. Ann N Y Acad Sci 1148, 495-503, 2008.

Karlen J, Ludvigsson J, Frostell A, Theodorsson E, Faresjo T. Cortisol in hair measured in young adults - a biomarker of major life stressors? BMC Clin Pathol 11, 12, 2011.

Kiazymov KhM, Buniatov MO, Mamedov ZM, Aliev RA. Determination of specific weight of the pigmented, grey and thick hairs from head. Georgian Med News 169, 78-81, 2009.

Kirschbaum C, Tietze A, Skoluda N, Dettenborn L. Hair as a retrospective calendar of cortisol production-Increased cortisol incorporation into hair in the third trimester of pregnancy. Psychoneuroendocrinology 34, 32-37, 2009.

Krumbholz A, Schonfelder M, Hofmann H, Thieme D. The plasma protein binding of the endogenous glucocorticosteroids is of vital importance for the concentrations in hair and saliva. Forensic Sci Int 286, 23-30, 2018.

Laudenslager ML, Jorgensen MJ, Grzywa R, Fairbanks LA. A novelty seeking phenotype is related to chronic hypothalamic-pituitary-adrenal activity reflected by hair cortisol. Physiol Behav 104, 291-295, 2011.

Meyer J, Novak M, Hamel A, Rosenberg K. Extraction and analysis of cortisol from human and monkey hair. J Vis Exp 83, e50882, 2014.

Nunes LA, Mussavira S, Bindhu OS. Clinical and diagnostic utility of saliva as a non-invasive diagnostic fluid: a systematic review. Biochem Med (Zagreb) 25, 177-192, 2015.

Pochigaeva K, Druzhkova T, Yakovlev A, Onufriev M, Grishkina M, Chepelev A, Guekht A, Gulyaeva N. Hair cortisol as a marker of hypothalamic-pituitary-adrenal Axis activity in female patients with major depressive disorder. Metab Brain Dis 32, 577-583, 2017.

Raul JS, Cirimele V, Ludes B, Kintz P. Detection of physiological concentrations of cortisol and cortisone in human hair. Clin Biochem 37, 1105-1111, 2004.

Skoluda N, Dettenborn L, Stalder T, Kirschbaum C. Elevated hair cortisol concentrations in endurance athletes. Psychoneuroendocrinology 37, 611-617, 2012.

Smy L, Shaw K, Amstutz U, Smith A, Berger H, Carleton B, Koren G. Hair cortisol as a hypothalamic-pituitary-adrenal axis biomarker in pregnant women with asthma: a retrospective observational study. BMC Pregnancy Childbirth 16, 176, 2016.

Wennig R. Potential problems with the interpretation of hair analysis results. Forensic Sci Int 107, 5-12, 2000.

Xiang L, Sunesara I, Rehm KE, Marshall GD Jr. A modified and cost-effective method for hair cortisol analysis. Biomarkers 21, 200-203, 2016. 